\title{
The analysis of acetaminophen (paracetamol) and seven metabolites in rat, pig and human plasma by U(H)PLC-MS
}

\author{
Rebecca Dargue ${ }^{1}$ (D), Isobelle Grant ${ }^{1}$, Leanne C Nye ${ }^{1}$, Andy Nicholls² (iD), Theo Dare ${ }^{2}$ (D)

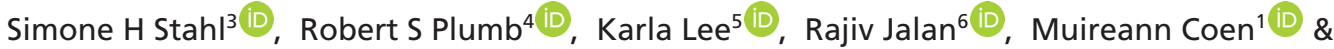 \\ Ian D Wilson*,1 (iD \\ ${ }^{1}$ Division of Systems Medicine, Department of Metabolism, Digestion \& Reproduction, Imperial College London, Exhibition Road, \\ South Kensington, London SW7 2AZ, UK \\ ${ }^{2}$ GlaxoSmithKline R\&D, Park Road, Ware, Hertfordshire SG12 ODP, UK \\ ${ }^{3}$ ADME Sciences, Clinical Pharmacology \& Safety Sciences, R\&D, AstraZeneca, Unit 310 Cambridge Science Park, Milton Road, \\ Cambridge CB4 OWG, UK \\ ${ }^{4}$ WatersCorporation, Milford, MA 01757, USA \\ ${ }^{5}$ Department of Clinical Science \& Services, Royal Veterinary College, University of London, Hertfordshire AL9 7TA, UK \\ ${ }^{6}$ UCL Institute for Liver \& Digestive Health, Royal Free Hospital, London NW3 2PF, UK \\ ${ }^{7}$ Oncology Safety, Clinical Pharmacology \& Quantitative Pharmacology, Clinical Pharmacology \& Safety Sciences, R\&D, \\ AstraZeneca, Cambridge CB4 OWG, UK \\ *Author for correspondence: i.wilson@imperial.ac.uk
}

A U(H)PLC-MS/MS method is described for the analysis of acetaminophen and its sulphate, glucuronide, glutathione, cysteinyl and $\mathrm{N}$-acetylcysteinyl metabolites in plasma using stable isotope-labeled internal standards. P-Aminophenol glucuronide and 3-methoxyacetaminophen were monitored and semiquantified using external standards. The assay takes $7.5 \mathrm{~min} / \mathrm{sample}$, requires only $5 \mu \mathrm{l}$ of plasma and involves minimal sample preparation. The method was validated for rat plasma and cross validated for human and pig plasma and mouse serum. LOQ in plasma for these analytes were $0.44 \mu \mathrm{g} / \mathrm{ml}$ (APAP-C), $0.58 \mu \mathrm{g} / \mathrm{ml}$ (APAP-SG), $0.84 \mu \mathrm{g} / \mathrm{ml}$ (APAP-NAC), $2.75 \mu \mathrm{g} / \mathrm{ml}$ (APAP-S), $3.00 \mu \mathrm{g} / \mathrm{ml}$ (APAP-G) and $16 \mu \mathrm{g} / \mathrm{ml}$ (APAP). Application of the method is illustrated by the analysis of plasma following oral administration of APAP to male Han Wistar rats.

First draft submitted: 10 January 2020; Accepted for publication: 23 March 2020; Published online: 28 April 2020

Keywords: acetaminophen $\bullet$ APAP $\bullet$ DILI $\bullet$ glutathione $\bullet$ metabolites $\bullet$ plasma analysis $\bullet$ UHPLC-MS

The analgesic and antipyretic drug acetaminophen (paracetamol, $N$-(4-hydroxyphenyl) acetamide, APAP) was launched over 60 years ago. It remains one of the most widely used drugs for the treatment of pain in the general human population. While acetaminophen, when used at recommended therapeutic doses (of up to $4 \mathrm{~g} /$ day) is generally considered to be safe, overdose results in half of all acute liver failure cases in the USA and the UK [1-5]. The overall mechanism responsible for the drug induced liver injury resulting from acetaminophen overdose is considered to be due to the inability of the major metabolic pathways of detoxication (glucuronidation and sulfation) to fully metabolize the drug. Thus, on overdose the bioactivation of APAP, via oxidative metabolism by cytochrome P450 2E1, leads to the formation of the highly reactive metabolite $N$-acetyl-p-benzoquinone imine (NAPQI) [6]. Once formed, NAPQI is usually detoxified by reaction with hepatic glutathione (GSH) but following GSH depletion, the metabolite reacts with cellular macromolecules eventually resulting in cell death [7]. As an important human drug for pain control, a hepatotoxin and an iconic model of reactive metabolite-induced drug induced liver injury, acetaminophen remains the subject of considerable research in humans and preclinical species. As a result numerous assays for acetaminophen in various biofluids, either alone or including variable numbers of metabolites, have been described to support both therapeutic monitoring or studies in animals. However, there remains a need for comprehensive, rapid and sensitive methods for the determination of the drug and its various conjugated 
and NAPQI-derived metabolites. While, providing sufficient sample is available, relatively unsophisticated and nonselective techniques, such as LC with UV detection, can be used for this purpose (e.g., [8-10]) the sensitivity and specificity of this approach is limited. Because of this, several LC-MS-based methods have been developed that offer the opportunity of reduced sample size and improved specificity compared with LC-UV [11-14]. For some of these LC-MS methods metabolite coverage was limited to the drug and its glucuronide and/or sulfate conjugates [11-13]. However, other LC-MS-based methods also provided coverage of the GSH-derived and other minor metabolites in both plasma and urine [14,15]. Recently, two further methods have been described offering validated HPLC [16] or UPLC [17]-ESI (electrospray ionization)-MS/MS methods that enable the quantification of APAP, its glucuronide (APAP-G), sulfate (APAP-S), GSH (APAP-GS), cysteinyl (APAP-C), N-acetylcysteinyl (APAP-NAC) [16,17] and methoxy-(APAP-OMe) [16] metabolites in human plasma. Here, we describe a 'fit for purpose' gradient reversed-phase U(H)PLC-MS/MS method for the quantification of APAP, APAP-S, APAP-G, APAP-C, APAP-NAC and APAP-GS metabolites, as well as the semi-quantitative monitoring of the APAP-OMe metabolite and the phenolic glucuronide of the $N$-deacetylated metabolite $p$-aminophenol (PAP-G). The method uses a minimal amount of sample and has been validated for rat plasma with cross validation to human and pig plasma. The method has also been used for mouse serum.

\section{Materials \& methods}

Chemicals \& reagents

APAP, APAP-G (sodium salt) and the deuterated internal standard APAP-d3 were purchased from Sigma Aldrich (Gillingham, UK), its sulfate (APAP-S, potassium salt), cysteinyl (APAP-C, trifluoroacetic acid salt), $\mathrm{N}$-acetylcysteinyl (APAP-NAC, disodium salt), GSH (APAP-SG, (disodium salt) and 3-methoxy (APAP-OMe) conjugates, and deuterated internal standards, APAP-S-d3 (potassium salt), APAP-G-d3 (sodium salt), APAP-C-d5 (TFA salt) APAP-NAC-d5 (sodium salt) and APAP-SG-d3 (disodium salt), were purchased from Toronto Research Chemicals (Toronto, Canada) and were used as supplied (salt conversion factors are provided in Supplementary Table 1). The metabolite PAP-G was purchased from SantaCruz Biotechnology (Dallas, TX, USA) and was used as supplied. Optima grade water was obtained from Fisher Scientific (Leicester, UK), LC-MS grade solvents and formic acid (FA) were from Sigma Aldrich. Control rat and human plasma for validation were obtained from SeraLabs (Haywards Heath, UK).

\section{Samples}

Rat plasma samples were obtained following a single oral administration of APAP at doses of $0,500 \mathrm{or} 1500 \mathrm{mg} / \mathrm{kg}$ (in $0.5 \%$ methylcellulose) to male Wistar Han rats (Crl:WI(Han)) (300-350 g). Rats were housed 3-5 per cage in polycarbonate solid bottom cages (Tecniplast, Kettering, UK) with Tapvei ${ }^{\circledR}$ Aspen Chips and Sizzle-Nest bedding and Tapvei ${ }^{\circledR}$ small Aspen bricks and polycarbonate tunnels as enrichment (all supplied by Dates and Machester, UK). Animals were fed with RM1 (E) SQC diet (Special Diets Service, Witham, UK) ad libitum, and were not fasted before APAP administration and had free access to $0.2 \mu \mathrm{M}$ filtered municipal water and food ad lib. Environmental controls were set to maintain conditions of $19-23^{\circ} \mathrm{C}$ and $55 \pm 15 \%$ relative humidity, with a 12-h light/dark cycle. Blood samples (ca. $800 \mu \mathrm{l}$ ) were collected (at 0, 1, 2, 4 and 8 from the tail vein (in-life) or a terminal sample from the vena cava at 24 -h postdose) into tubes containing lithium heparin. Plasma samples $(200 \mu \mathrm{l})$ were obtained by centrifugation at $1200 \mathrm{~g}\left(4^{\circ} \mathrm{C}\right.$ ), with the samples then frozen and stored at $-70^{\circ} \mathrm{C}$ (or lower) until analysis. Animals were sacrificed using halothane at the end of the studies. The study was performed by Drug Safety and Metabolism, AstraZeneca UK (all experiments were conducted in compliance with UK home office licences issued under the UK Animals [Scientific Procedures] Act 1986 after review by the local Ethics Committee).

\section{Standard curve \& quality control preparation}

Stock solutions for the preparation of calibration curves and quality control (QC) samples (including low [LQC], mid [MQC], high [HQC] and the [LLOQ], [ULOQ]) for APAP and APAP metabolite standards were prepared from ca. $1 \mathrm{mg} / \mathrm{ml}$ solutions in $\mathrm{MeOH}$. These solutions were then diluted to concentrations of $500 \mu \mathrm{g} / \mathrm{ml}$ for APAP, $93.7 \mu \mathrm{g} / \mathrm{ml}$ for APAP-G, $85.9 \mu \mathrm{g} / \mathrm{ml}$ for APAP-S, $18.2 \mu \mathrm{g} / \mathrm{ml}$ for APAP-SG, $17.5 \mu \mathrm{g} / \mathrm{ml}$ for APAP-NAC and $13.9 \mu \mathrm{g} / \mathrm{ml}$ for APAP-C (see Supplementary Table 2) with subsequent dilutions to prepare standard curve and QC solutions (Supplementary Tables 2-4). An internal standard (IS) stock solution (see Supplementary Table 5) was also prepared at concentrations of $500 \mu \mathrm{g} / \mathrm{ml}$ for APAP-d $3,94.3 \mu \mathrm{g} / \mathrm{ml}$ for APAP-G-d3, $86.0 \mu \mathrm{g} / \mathrm{ml}$ for APAP-S-d3, $28.1 \mu \mathrm{g} / \mathrm{ml}$ for APAP-NAC-d 5 and $21.0 \mu \mathrm{g} / \mathrm{ml}$ for APAP-C-d5. For both calibration and QC 


\begin{tabular}{|c|c|c|c|c|c|}
\hline APAP & APAP-G & APAP-S & APAP-C & APAP-SG & APAP-NAC \\
\hline 24 & 4.50 & 4.12 & 0.67 & 0.88 & 0.84 \\
\hline 40 & 7.50 & 6.87 & 1.11 & 1.46 & 1.40 \\
\hline 60 & 11.2 & 10.3 & 1.67 & 2.19 & 2.10 \\
\hline 150 & 28.1 & 25.8 & 4.173 & 5.47 & 5.26 \\
\hline 250 & 46.9 & 42.9 & 6.94 & 9.12 & 8.77 \\
\hline 500 & 93.7 & 85.9 & 13.9 & 18.2 & 17.5 \\
\hline
\end{tabular}

The concentrations given in this table are those in the final samples following sample preparation for analysis which results in a 1000-fold dilution compared with the original sample.

\begin{tabular}{|c|c|c|c|c|c|c|}
\hline QC & APAP & APAP-G & APAP-S & APAP-C & APAP-SG & APAP-NAC \\
\hline ULOQ & 500 & 93.7 & 85.9 & 13.9 & 18.2 & 17.5 \\
\hline Mid & 100 & 18.7 & 17.2 & 2.78 & 3.65 & 3.51 \\
\hline Low & 48 & 9.00 & 8.24 & 1.33 & 1.75 & 1.68 \\
\hline LLOQ & 16 & 3.00 & 2.75 & 0.44 & 0.58 & 0.84 \\
\hline
\end{tabular}

The concentrations given in this table are those in the final samples following sample preparation for analysis which results in a 1000 -fold dilution compared with the original sample.

samples, $5 \mu \mathrm{l}$ of pooled blank matrix from either human, rat, mouse or pig as appropriate, were mixed with $35 \mu \mathrm{l}$ of methanol, $10 \mu \mathrm{l}$ of IS stock solution and $50 \mu \mathrm{l}$ of the relevant standard stock solution. For single blanks, $5 \mu \mathrm{l}$ of plasma were mixed with $85 \mu \mathrm{l}$ of $\mathrm{MeOH}$ and $10 \mu \mathrm{l}$ of IS stock solution. Double blanks consisted of $5 \mu \mathrm{l}$ of blank matrix mixed with $95 \mu \mathrm{l}$ of $\mathrm{MeOH}$. All samples were kept at $-20^{\circ} \mathrm{C}$ for $20 \mathrm{~min}$ to precipitate proteins, and then centrifuged for $10 \mathrm{~min}$ at $10,000 \mathrm{~g}$. A $20 \mu \mathrm{l}$ aliquot of the clear supernatant from each sample was added to $980 \mu \mathrm{l}$ of water in glass vials for analysis. Final concentrations of the IS's were; APAP-d3: $200 \mathrm{ng} / \mathrm{ml}$, APAP-G-d3: $37.7 \mathrm{ng} / \mathrm{ml}$, APAP-S-d3: $34.4 \mathrm{ng} / \mathrm{ml}$, APAP-NAC-d5: $11.2 \mathrm{ng} / \mathrm{ml}$ and APAP-C-d5: $8.38 \mathrm{ng} / \mathrm{ml}$, APAP-SG-d3: $11.0 \mathrm{ng} / \mathrm{ml}$ (APAP-SG-d3 only became available part way though the study did not form part of the initial 3-day validation but was added in subsequently).

The final concentrations of the analytes in both calibration curves and QC samples following sample preparation in rat plasma are given in Tables 1 and 2 and reflect the overall dilution by 1 in 1000 required to bring them onto the linear range of the mass spectrometer. For cross validation to human plasma, the same concentration ranges were used for QC's and standard curves.

Based on a preliminary evaluation of porcine samples, PAP-G was added to stock solutions at a concentration of $500 \mu \mathrm{g} / \mathrm{ml}$ and the concentration of APAP-G in the solution was increased to $703 \mu \mathrm{g} / \mathrm{ml}$ (see Supplementary Table 16). In the case of cross validation to mouse serum concentrations were as for rat, with the addition of APAP-OMe at $20 \mu \mathrm{g} / \mathrm{ml}$ (see Supplementary Table 20 )for further details. For determination of freeze-thaw and bench-top stability, a sample was prepared in plasma containing the standard stock solutions in methanol at $<5 \%$ of the total sample volume. Methanolic solutions of APAP $(10 \mathrm{mg} / \mathrm{ml})$ APAP-G $(2.34 \mathrm{mg} / \mathrm{ml})$, APAP-S $(2.15 \mathrm{mg} / \mathrm{ml})$, APAP-C $(0.694 \mathrm{mg} / \mathrm{ml})$, APAP-NAC $(0.877 \mathrm{mg} / \mathrm{ml})$ and APAP-SG $(0.912 \mathrm{mg} / \mathrm{ml})$ were prepared with $5 \mu \mathrm{l}$ of the APAP solution, $4 \mu \mathrm{l}$ each of the APAP-S and APAP-G and $2 \mu \mathrm{l}$ each of APAP-C, -NAC and -SG solutions added to plasma to create a total volume of $0.5 \mathrm{ml}$. This was then further diluted $5 \times$ in plasma to produce a plasma stock sample. IS stock solution was prepared as stated previously then diluted 2.5-times in MeOH. The plasma stock sample was prepared by diluting it with $40 \mu \mathrm{l}$ of $\mathrm{MeOH}$ and $5 \mu \mathrm{l}$ of IS solution, giving the prepared sample a concentration equivalent to the MQC.

\section{Sample preparation}

Samples $(5 \mu \mathrm{l})$, from either human, rat, or pig plasma, as appropriate, were mixed with and IS stock solution $(10 \mu \mathrm{l})$ and $\mathrm{MeOH}(85 \mu \mathrm{l})$, briefly vortexed then kept at $-20^{\circ} \mathrm{C}$ for $20 \mathrm{~min}$ before centrifugation $(10 \mathrm{~min}, 10,000 \mathrm{~g})$ to 
Table 3. Measured concentration ranges of APAP and metabolites in rat plasma samples.

\begin{tabular}{lllllll} 
APAP $\mu \mathrm{g} / \mathrm{ml}$ & APAP-G $\mu \mathrm{g} / \mathrm{ml}$ & APAP-S $\mu \mathrm{g} / \mathrm{ml}$ & APAP-C $\mu \mathrm{g} / \mathrm{ml}$ & APAP-SG $\mu \mathrm{g} / \mathrm{ml}$ & APAP-NAC $\mu \mathrm{g} / \mathrm{ml}$ \\
$16-500$ & $3.00-93.7$ & $2.75-85.9$ & $0.44-13.9$ & $0.58-18.2$ & $0.84-17.5$ \\
\hline
\end{tabular}

\section{Table 4. MS and LC data for APAP and metabolites quantified/monitored in plasma.}

\begin{tabular}{|c|c|c|c|c|c|}
\hline Compound & Parent ion $(m / z)$ & $\begin{array}{l}\text { Product ion for } \\
\text { quantification }(\mathrm{m} / \mathrm{z})\end{array}$ & Cone voltage (V) & Collision voltage (V) & $\mathrm{RT}$ (min) \\
\hline APAP & 152.1 & 110.1 & 30 & 16 & 2.91 \\
\hline APAP-D3 & 155.1 & 110.9 & 30 & 20 & 2.89 \\
\hline APAP-C & 271.0 & 139.9 & 34 & 24 & 2.53 \\
\hline APAP-C D5 & 276.2 & 142.8 & 34 & 26 & 2.50 \\
\hline APAP-S & 232.1 & 110.1 & 30 & 22 & 2.17 \\
\hline APAP-S D3 & 235.0 & 111.0 & 30 & 22 & 2.15 \\
\hline APAP-G & 328.1 & 152.1 & 20 & 14 & 1.79 \\
\hline APAP-G D5 & 353.2 & 177.1 & 42 & 16 & 1.77 \\
\hline APAP-NAC & 335.1 & 152.0 & 6 & 16 & 4.98 \\
\hline APAP-NAC-D5 & 340.2 & 152.0 & 34 & 18 & 4.96 \\
\hline APAP-SG & 457.2 & 139.9 & 30 & 36 & 3.85 \\
\hline APAP-SG D3 & 460.266 & 331.076 & 32 & 14 & 3.81 \\
\hline PAP-G & 308.33 & 199.05 & 2.0 & 12 & 0.37 \\
\hline APAP-OMe & 182.196 & 108.08 & 16 & 20 & 4.04 \\
\hline
\end{tabular}

remove precipitated proteins. Then, $20 \mu \mathrm{l}$ of the clear supernatant was mixed with $980 \mu \mathrm{l}$ of water in glass vials for analysis (meaning that samples underwent a 1000-fold dilution before analysis). The concentration ranges measured within the plasma samples are given in Table 3.

\section{U(H)PLC-MS}

Chromatography was performed on an Acquity U(H)PLC system using a $2.1 \times 100 \mathrm{~mm} 1.8 \mu \mathrm{m} 130$ A C18 ACQUITY HSS T3 column (Waters Corporation, Manchester, UK) with a multilinear reversed-phase gradient. The mobile phases consisted of water and $0.1 \%(\mathrm{v} / \mathrm{v}) \mathrm{FA}$ (solvent A) and $\mathrm{MeOH}$ and $0.1 \%(\mathrm{v} / \mathrm{v}) \mathrm{FA}$ (solvent B). The gradient was performed over $7.5 \mathrm{~min}$ at a flow rate of $0.6 \mathrm{ml} / \mathrm{min}$ at $40^{\circ} \mathrm{C}$ with the starting conditions set at $5 \%$ solvent B for $0.5 \mathrm{~min}$, increasing linearly to $7 \%$ by $1.85 \mathrm{~min}$ then to $8 \%$ by $1.9 \mathrm{~min}$, then $10 \%$ by $2.5 \mathrm{~min}, 16 \%$ by $4.0 \mathrm{~min}, 25 \%$ by $5 \mathrm{~min}$ increasing rapidly to $95 \%$ by $5.1 \mathrm{~min}$ to wash the column. The solvent composition was held at $95 \%$ B for 0.9 min before returning to $5 \%$ B at $6.1 \mathrm{~min}$ for re-equilibration (1.4 min). The resulting analysis time was $7.5 \mathrm{~min} /$ sample. In between samples, the sample loop was subject to both weak and strong washes of 90:10 water/ $\mathrm{MeOH}(\mathrm{v} / \mathrm{v})$ and $100 \%$ isopropanol, respectively.

MS/MS data were acquired using a Xevo tandem quadruple (TQ-S) mass spectrometer (Waters Corporation). The quantification of the analytes was performed using MS/MS in positive ESI mode with multiple reaction monitoring optimized for the individual analytes. The appropriate MS conditions were determined for each compound by direct infusion. For MS, the desolvation gas was nitrogen, and the collision gas employed was argon. Additional conditions included a capillary voltage of $3 \mathrm{kV}$, a source offset of $30 \mathrm{~V}$, a desolvation temperature of $500^{\circ} \mathrm{C}$, a source temperature of $150^{\circ} \mathrm{C}$. The desolvation gas flow was $1000 \mathrm{l} / \mathrm{h}$ with a cone gas flow of $150 \mathrm{l} / \mathrm{h}$. The nebulizer gas was set at 7.0 bar and the collision gas at $0.13 \mathrm{ml} / \mathrm{min}$. Table 4 for individual parameters for each compound.

For analysis, $2 \mu \mathrm{l}$ of a double blank sample were injected first in order to confirm system cleanliness. Then, $2 \mu \mathrm{l}$ of the single blank was injected, followed by further $2 \mu \mathrm{l}$ samples of the calibration curve solutions (increasing in concentration from low to high). These samples were followed by the double blank. Following these injections, the study samples were analyzed, which had been randomized before protein precipitation so as to reduce any analytical bias. The QC samples (at least six with a minimum of two at each of the LQC, MQC and HQC concentrations) were analyzed at regular intervals interspersed evenly among the study samples throughout the course of the run. Following analysis of the study samples and QCs, a second set of calibration samples were also analyzed. The analytical run sequence is summarized in Figure 1. 


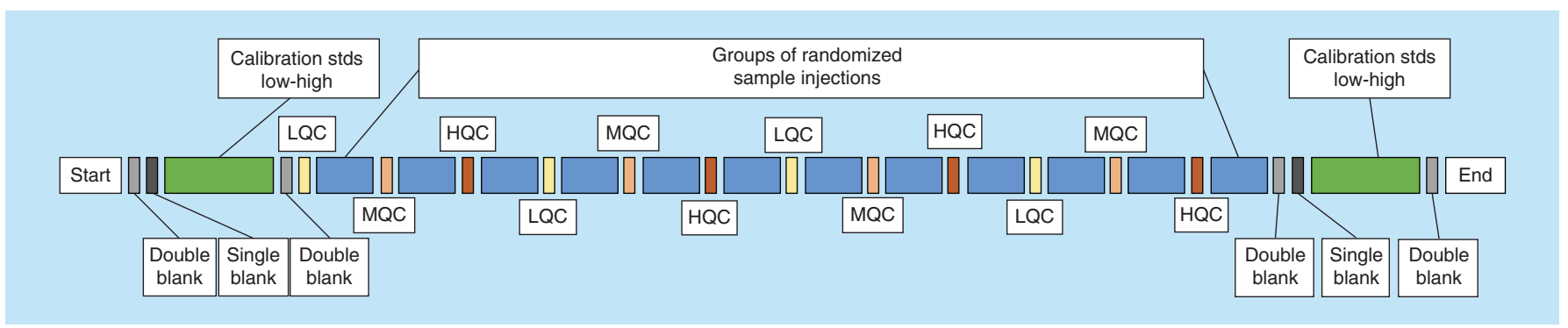

Figure 1. Sequence of analysis for APAP/metabolite quantification of randomized samples bracketed by calibration standards and interspersed with QC injections.

QC: Quality control.

\section{Method validation}

Method validation was based on the recommendations contained in the US FDA guidance for industry on bioanalytical methods [18]. A 3-day validation was undertaken for rat plasma and the resulting method was then cross validated to human and pig plasma as described below.

\section{Linearity}

Linearity was determined from the evaluation of calibration curves generated from calibration standards with acceptable deviation ( $\leq 15 \%$ over the range of the standard curve and $\leq 20 \%$ at the LLOQ) from their nominal values, using least squares linear regression with weighting $1 / \mathrm{x}$ or $1 / \mathrm{x}^{2}$ for APAP-SG. Linearity was assessed using the $\mathrm{R}^{2}$ correlation coefficient, which was required to be $>0.99$ over the 3 days of the validation.

\section{Precision \& accuracy}

Assay precision was determined by the analysis of six replicates at each of the concentrations of the LLOQ QC, LQC, MQC, HQC and ULOQ QC samples on all 3 days of the full validation for rat plasma and 1 day for each of the cross validation studies. The intra-assay precision was determined using the CV of the six replicates on 1 day. The interassay precision was determined as the CV of each set of QC samples over three batches $(\mathrm{n}=18)$ at each QC concentration. The interassay accuracy was described by the mean deviation of the QCs over 3 days $(\mathrm{n}=18)$ at each QC concentration. For both intra- and interassay assessments, the acceptable CV was set at $\leq 15 \%$ for all QCs except the LLOQ QC which was set at $\leq 20 \%$. A minimum of two thirds of the QCs were required to fall within these limits for acceptance.

\section{Carryover}

Carryover was assessed with a double blank run immediately after an ULOQ calibration standard and was considered acceptable if the response for any of the analytes was $\leq 20 \%$ of the average response of the LLOQ standards. Carryover for the IS's was deemed acceptable if the response for the double blank sample was $\leq 5 \%$ of the average response from the acceptable calibration standards (including the single blank).

\section{Recovery}

All analytes and ISs were spiked into six individual lots of blank plasma, both before and after protein precipitation, at both the LQC and HQC concentrations. Peak areas of analytes at the LQC or HQC spiked in before extraction were compared with those from analytes spiked in after extraction, calculated as a percentage recovery.

\section{Matrix effects}

Matrix effects were assessed by spiking ISs and standards for each analyte into six blank matrix samples of plasma after protein precipitation, with six replicates at the concentration of the LQC and six at the concentration of the HQC (referred to as over-spiked samples). Reference solutions were prepared by spiking ISs and standards into water to reflect the LQC and HQC concentrations. Matrix effects were calculated by comparing the peak area for each standard or IS in the reference solution to the over-spiked samples. The IS normalized matrix factor was calculated by dividing the matrix factor calculated from the unlabeled standard by the matrix factor calculated from 
the labeled IS. To be acceptable the CV of the IS normalized matrix factor at each concentration had to be $\leq 15 \%$ at both LQC and HQC concentrations.

\section{Selectivity \& specificity}

The method was evaluated for selectivity by assessing interference from the matrix in blank analyte free matrix (double blank samples), and also for selectivity between analytes and ISs by analyzing blank matrix samples containing individual metabolites or ISs only. Six double blanks containing none of the analytes were processed. Interference was defined as any response at the retention times of the analytes with a response $\geq 20 \%$ of the mean LLOQ response. For IS, interference was defined as a response at the retention time of the IS with a response $\geq 5 \%$ of the average IS response in the calibration curve. To determine IS and analyte selectivity, three aliquots of the same lot of blank plasma were spiked with IS only or individual analytes at the ULOQ only. Interference with another analyte was defined as any response at the retention time of the analyte with a peak area $\geq 20 \%$ of the average LLOQ response. Any responses at the retention time of an IS in the individual analyte samples were considered interference if found to be $\geq 5 \%$ of the average IS response for that IS.

\section{Stability of samples \& solutions \\ Freeze-thaw stability}

For investigation of freeze-thaw stability, a 'bulk' plasma sample was prepared with standards added to blank rat plasma at $<5 \%$ of the total volume (i.e., $50 \mu \mathrm{l}$ of stock solutions added to $950 \mu \mathrm{l}$ blank plasma). Further dilutions of this sample were performed in plasma to produce a bulk sample with concentrations of analytes at those of the MQC sample. Aliquots $(5 \mu \mathrm{l})$ of this MQC bulk plasma were taken and either analyzed immediately (time $0 \mathrm{~h})$ or placed in the freezer at $-40^{\circ} \mathrm{C}$ degrees for half an hour, then thawed at room temperature at regular intervals for up to six freeze-thaw cycles. For each cycle, six replicate samples were analyzed. Stability was calculated as the percentage peak area compared with the six MQC samples prepared with no freeze-thaw cycles.

\section{Benchtop stability}

The stability of standards in solution at ambient temperature was measured using the 'bulk prepared' MQC plasma sample as above, with plasma samples placed on the bench at ambient temperature for $4 \mathrm{~h}$, and then analyzed. Analyte stability was calculated as a percentage of the responses for the MQC samples prepared at $0 \mathrm{~h}$.

\section{Autosampler stability}

To assess the stability of the analytes over the course of the analysis, six replicates of each QC were analyzed, then left for $36 \mathrm{~h}$ in the autosampler at $5^{\circ} \mathrm{C}$ and then re-assayed against a fresh calibration curve and the $\mathrm{CV}$ and bias calculated.

\section{Dilution integrity}

A dilution integrity QC (DIQC) sample was prepared at $3 \times$ the concentration of the ULOQ. 6 aliquots of the DIQC were diluted 1:10 to give 6 diluted samples (100 $\mu \mathrm{l}$ of DIQC into $900 \mu \mathrm{l}$ of water). The diluted DIQCs were then analyzed as part of the validation and quantified taking into account the 1 in 10 dilution of the IS. In order to be acceptable, at least four of the six DIQCs were required to be $\leq \pm 15 \%$ of the nominal concentration with a CV for the six QC samples of $\leq 15 \%$.

\section{Data analysis}

The raw LC-MS/MS data were processed by the TargetLynx application package within MassLynx software (Waters Corporation). The raw data were mean smoothed and peak integration was performed using the ApexTrak algorithm.

\section{Results \& discussion \\ Chromatography}

Optimization of the chromatographic conditions for the reversed-phase gradient resulted in the separation of APAP and its metabolites shown in Figure 2. The sensitivity of the assay proved to be sufficiently high that only $5 \mu \mathrm{l}$ of plasma were required in order to perform the analysis with sample preparation limited to protein precipitation using methanol followed by dilution with water for injection. 


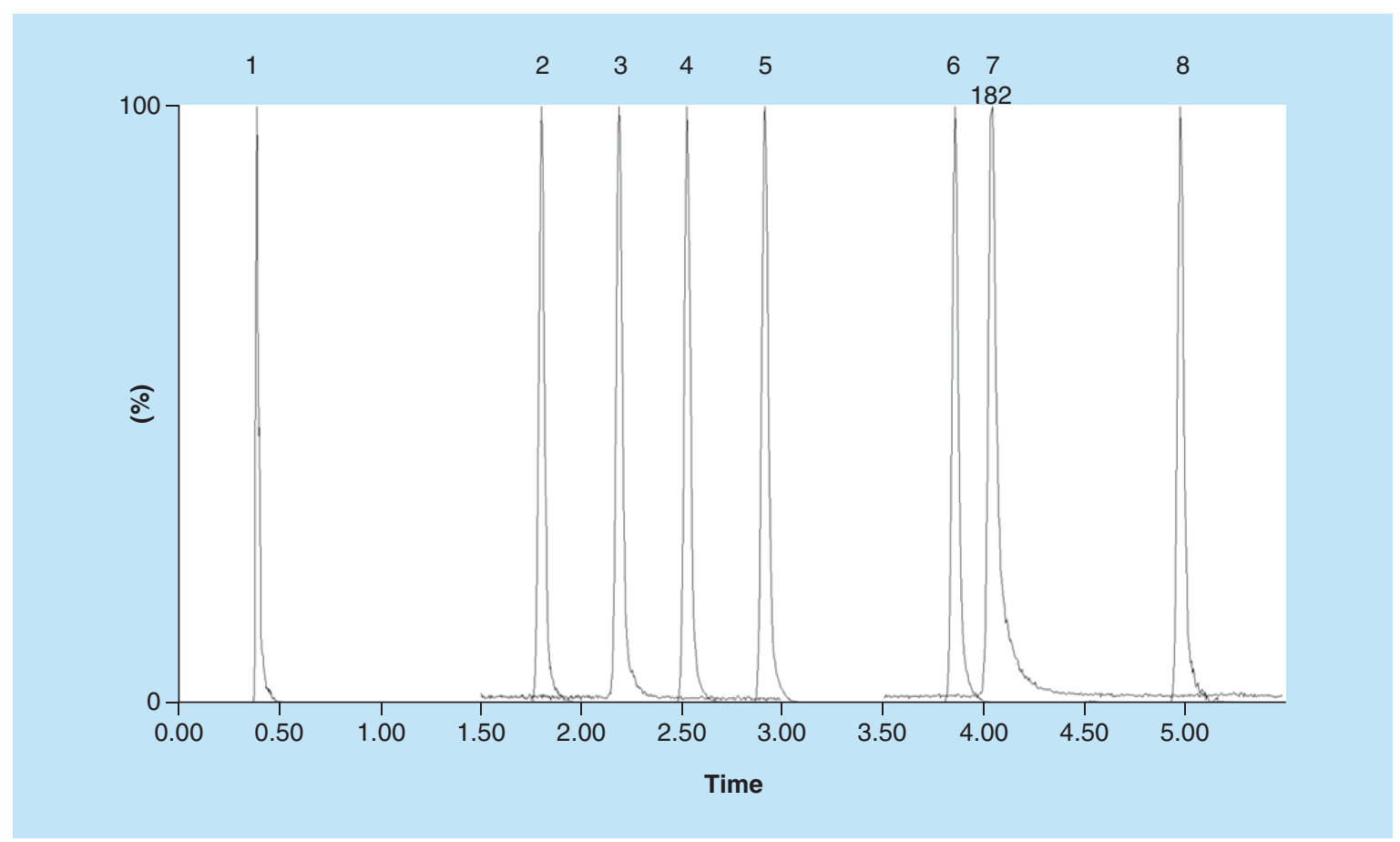

Figure 2. A reconstructed ion mass chromatogram for the standards of APAP and the targeted metabolites. 1 = PAP-G 2 = APAP-G, 3 = APAP-S, 4 = APAP-C, 5 = APAP, 6 = APAP-SG, 7 = APAP-OMe and 8 = APAP-NAC obtained using the optimized chromatographic system.

Based on this combination of sample preparation and chromatographic separation, the method was validated for rat plasma with intra (within day) and interday (3 days) accuracy and precision assessed for the analysis of APAP, and the five metabolites APAP-S, APAP-G, APAP-C, APAP-NAC and APAP-SG targeted for quantification. Over the 3 days of the interday validation, the method was found to be linear over the concentration ranges measured (see Table 1) for all of the analytes with all $\mathrm{r}^{2}$ values above 0.99 (Table 5, for equations of the line see Supplementary Table 6). In addition, factors such as the LLOQ, linearity, recovery, selectivity, matrix effects and carryover were also determined for all of the compounds assayed (see Table 5 for a summary of the validation data).

\section{Precision \& accuracy}

For each day, and across all 3 days, the CV and bias data for QCs met the acceptance criteria outlined in the methods section; these, and other, validation data (e.g., stability, recovery dilution integrity etc.) are summarized in Table 5 and full results for the validation are provided in Supplementary Tables 7-10. The resulting LLOQs were $16 \mu \mathrm{g} / \mathrm{ml}$ for APAP, $3.00 \mu \mathrm{g} / \mathrm{ml}$ for APAP-G, $2.75 \mu \mathrm{g} / \mathrm{ml}$ for APAP-S, $0.84 \mu \mathrm{g} / \mathrm{ml}$ for APAP-NAC, $0.58 \mu \mathrm{g} / \mathrm{ml}$ for APAP-SG and $0.44 \mu \mathrm{g} / \mathrm{ml}$ for APAP-C. The corresponding ULOQs were $500 \mu \mathrm{g} / \mathrm{ml}$ for APAP, $93.7 \mu \mathrm{g} / \mathrm{ml}$ for APAP-G, $85.9 \mu \mathrm{g} / \mathrm{ml}$ for APAP-S, $18.2 \mu \mathrm{g} / \mathrm{ml}$ for APAP-SG, $17.5 \mu \mathrm{g} / \mathrm{ml}$ for APAP-NAC and $13.9 \mu \mathrm{g} / \mathrm{ml}$ for APAP-C. In terms of the absolute amounts of each analyte (as opposed to back-calculated sample concentrations of $\mu \mathrm{g} / \mathrm{ml}$ of sample), these values corresponded to quantities of $0.89 \mathrm{pg}$ (APAP-C), $1.17 \mathrm{pg}$ (APAP-SG), $1.68 \mathrm{pg}$ (APAP-NAC), $5.49 \mathrm{pg}$ (APAP-S), $6.00 \mathrm{pg}$ (APAP-G) and $32 \mathrm{pg}$ (APAP) actually injected on column.

\section{Matrix interference \& recovery}

The results obtained for the determination of the matrix factors for the various analytes with isotopically labeled IS's were all acceptable, with CVs below 15\% (summarized in Table 5).

In the case of APAP-OMe, matrix effects were highly variable with CVs for the HQC and LQCs of 36 and 18\%, respectively, confirming the semi-quantitative nature of the assay for this metabolite. Recoveries were over $80 \%$ for all analytes except APAP-NAC for the LQC, where the mean recovery was 78.7\%, with CVs generally below $10 \%$. These values were similar to those reported for other assays for these analytes [14-17]. The recovery and matrix factor data for each of the analytes are summarized in Table 5. 
Table 5. Summary of intra and 3-day interday data for the method validation in rat plasma.

\begin{tabular}{|c|c|c|c|c|c|c|c|}
\hline Parameter & QC Level & APAP & APAP-S & APAP-G & APAP-C & APAP-NAC & APAP-SG \\
\hline Linearity $\left(\right.$ mean $\left.R^{2}\right) n=3$ & - & 0.995 & 0.997 & 0.994 & 0.995 & 0.995 & 0.993 \\
\hline \multirow{5}{*}{$\begin{array}{l}\text { Intraday }{ }^{\ddagger} \text { accuracy (mean } \\
\% \text { bias), } \mathrm{n}=6\end{array}$} & LLOQ & 0.1 & 2.6 & -13.5 & 4.2 & 5.9 & 9.4 \\
\hline & LQC & -5.9 & 0.6 & -12.2 & -2.1 & -2.1 & -3.6 \\
\hline & MQC & 4.3 & 3.6 & 0.3 & -2.5 & 2.1 & -5.8 \\
\hline & HQC & 0.7 & 3.4 & -1 & 1.5 & -0.9 & 0.3 \\
\hline & ULOQ & 3.3 & 6.8 & 1.9 & 6.3 & 5.7 & 1.9 \\
\hline \multirow{5}{*}{$\begin{array}{l}\text { Interday accuracy (mean } \\
\% \text { bias), } n=18\end{array}$} & LLOQ & 1.8 & -8.6 & 0.6 & -1.1 & -1.1 & -7.5 \\
\hline & LQC & 0.9 & -1.8 & 8.6 & -1.2 & 3.3 & -0.5 \\
\hline & MQC & -3.4 & -0.3 & 6 & -0.4 & 0.6 & -0.6 \\
\hline & HQC & 1.4 & -0.8 & 6.8 & -2.9 & 1.4 & -5 \\
\hline & ULOQ & 1.7 & -2.3 & 6.6 & -4.9 & 0.9 & -6.1 \\
\hline \multirow{5}{*}{$\begin{array}{l}\text { Intraday precision } \\
(\mathrm{CV}), \mathrm{n}=6\end{array}$} & LLOQ & 4.8 & 13.4 & 7.4 & 7.1 & 8.8 & 8.2 \\
\hline & LQC & 12.2 & 7.5 & 11.8 & 6.2 & 6.2 & 6.8 \\
\hline & MQC & 8.7 & 10 & 8.7 & 8.8 & 10.4 & 9 \\
\hline & HQC & 4.9 & 3.4 & 5 & 3.7 & 6.1 & 6.6 \\
\hline & ULOQ & 5.6 & 7.1 & 5.6 & 6.8 & 7.4 & 3.7 \\
\hline \multirow{5}{*}{$\begin{array}{l}\text { Interday precision (CV), } \\
\mathrm{n}=18\end{array}$} & LLOQ & 6.8 & 17.5 & 15.9 & 11.1 & 12.5 & 8.7 \\
\hline & LQC & 10 & 12.8 & 8.7 & 8.2 & 11.3 & 7.5 \\
\hline & MQC & 8.5 & 10.2 & 8.8 & 7.5 & 9.6 & 9.5 \\
\hline & HQC & 4.9 & 5.8 & 6.7 & 5.8 & 7.8 & 8.3 \\
\hline & ULOQ & 5.3 & 6.9 & 8.3 & 5.6 & 7.6 & 5.9 \\
\hline \multirow{4}{*}{$\begin{array}{l}\text { Matrix factor } \\
(n=6 ; \text { corrected using IS } \\
\text { except for APAP-SG) }\end{array}$} & LQC (\%) & 99 & 102 & 101 & 99 & 110 & $105^{\dagger}$ \\
\hline & $\mathrm{CV}(\mathrm{n}=6)$ & 2.47 & 4.08 & 6.14 & 5.13 & 4.73 & - \\
\hline & HQC (\%) & 93 & 94 & 89 & 95 & 90 & 110 \\
\hline & $\mathrm{CV}(n=6)$ & 3.11 & 5.59 & 3.89 & 2.26 & 6.92 & - \\
\hline \multirow[t]{4}{*}{ Recovery } & LQC (\%) & 94.6 & 90.8 & 87.8 & 88.3 & 78.7 & 105 \\
\hline & $C V(n=6)$ & 2.76 & 8.02 & 8.56 & 14.3 & 17.5 & 11.4 \\
\hline & HQC (\%) & 88.0 & 86.4 & 82.6 & 88.7 & 81.4 & 81.5 \\
\hline & $\mathrm{CV}(\mathrm{n}=6)$ & 9.59 & 7.8 & 10.6 & 5.72 & 4.23 & 5.57 \\
\hline \multirow{2}{*}{$\begin{array}{l}\text { DIQC dilution integrity } \\
(n=6)\end{array}$} & Mean $\%$ bias & -10.3 & -13.3 & -8.67 & -10.6 & -13.9 & 12.2 \\
\hline & $\mathrm{CV}$ & 1.52 & 5.94 & 3.1 & 6.84 & 21 & 4.37 \\
\hline \multirow{5}{*}{$\begin{array}{l}36 \mathrm{~h} \text { autosampler stability } \\
\text { (\% bias } \pm \mathrm{CV}, \mathrm{n}=6 \text { ) }\end{array}$} & LLOQ & $-9.06 \pm 6.71$ & $-7.54 \pm 5.54$ & $4.92 \pm 3.39$ & $5.71 \pm 3.93$ & $-15.4 \pm 11.8$ & $-5.13 \pm 3.72$ \\
\hline & LQC & $-14.8 \pm 11.3$ & $-13.5 \pm 10.2$ & $-19.7 \pm 15.5$ & $-10.9 \pm 8.16$ & $-7 \pm 5.13$ & $-5.31 \pm 3.86$ \\
\hline & MQC & $-16 \pm 12.3$ & $-14.5 \pm 11$ & $-9.18 \pm 6.8$ & $-11.5 \pm 8.66$ & $-10.1 \pm 7.48$ & $-7.14 \pm 5.24$ \\
\hline & HQC & $-14.9 \pm 11.4$ & $-17.3 \pm 13.4$ & $-15.9 \pm 12.2$ & $-12.6 \pm 9.5$ & $-13.7 \pm 10.4$ & $-8.63 \pm 6.37$ \\
\hline & ULOQ & $-15 \pm 11.5$ & $-18.8 \pm 14.7$ & $-12.6 \pm 9.48$ & $-12 \pm 8.99$ & $-10.6 \pm 7.91$ & $-12.4 \pm 9.32$ \\
\hline
\end{tabular}

Stability

Previous studies have reported varying results for stability [14-17] and, while in general, APAP and the major conjugated metabolites APAP-G and APAP-S were found to be stable, this was less clear cut for the other metabolites. Therefore, analyte stability was reinvestigated here with respect to freeze-thaw, ambient temperature ('bench top') and 36-h autosampler stability. 


\section{Freeze-thaw \& ambient temperature stability}

The effects of up to six freeze-thaw cycles (Supplementary Tables $11 \& 12$ ) did not indicate any major instability in any of the analytes such that, while overall there may have been a modest decline, there was no discernible trend.

Similarly, in the case of stability on the bench at ambient temperature, there was little evidence of a trend in peak areas and all analytes appeared to be stable for $4 \mathrm{~h}$ on the bench (the maximum time tested) (Supplementary Table 13).

\section{Autosampler stability}

Previous studies have reported varying results for stability, with some reports showing all analytes to be stable in the autosampler for up to $48 \mathrm{~h}[14,16]$, while another report found that APAP-SG was only stable for up to $24 \mathrm{~h}$, whereas the other analytes remained stable for up to $48 \mathrm{~h}$ [17]. Another study found all metabolites to be stable in the autosampler for up to $73 \mathrm{~h}$ (but also stated that APAP-SG degraded to APAP-C in human plasma) [15]. Given the differences seen in analyte stability in earlier methods, we studied the analytes under our autosampler conditions which we felt may not have exactly replicated the conditions used in previous validations. This was done using QC samples kept in the autosampler for $36 \mathrm{~h}\left(\right.$ at $5^{\circ} \mathrm{C}$ ) and analyzed against a freshly prepared standard curve. This study indicated a small overall decrease in response for all analytes (Table 5 and Supplementary Table 14). The percentage decline (bias) for most analytes was generally below 15\%, with APAP-S and APAP-G showing a limited number of values between 15 and $20 \%$ (see Table 5). This suggests that processed samples should not be left for an extended period in the autosampler (or indeed the fridge). The implication is also clear that extended analytical runs may pose an analytical risk. With respect to stability over a shorter time period, we suggest that, in practice, for a typical rat toxicology study (as exemplified here), the analytes were stable over the ca. 15-h duration of the analysis as no time-dependent change in the response of the QCs or the standard curves were noted. However, had the run failed (because e.g., of an instrumental failure of some sort), our stability data would have indicated that simply rerunning the samples after the problem had been rectified might have been problematic, with complete reanalysis indicated. Pragmatically, for fit for purpose methods, we therefore suggest that it may be possible to perform 'in use' stability testing by looking for trends in declining response in the QC samples and standard curve data.

\section{Dilution integrity}

Dilution integrity was determined by quantification of the analytes following the tenfold dilution of a (DIQC) sample prepared at three-times the concentration of the ULOQ as described in the experimental methods section. The analysis of this sample showed that the results of the serial dilution met the acceptance criteria of being within $\pm 15 \%$ of the nominal concentration (with a CV of $\leq 15 \%$ ) for all analytes with the exception of APAPNAC. This is unlikely to present a problem in practice as concentrations of APAP-NAC are generally low in plasma and serum meaning that the need for sample dilution is remote. Results for the analyses of the DIQC integrity sample are summarized in Table 5 and Supplementary Table 15.

\section{Carryover, selectivity \& specificity}

Carryover was low, and within the acceptance criteria with responses at the retention times of the individual compounds below 20\% of the LLOQ for all analytes and 5\% of the IS responses (see Supplementary Tables 16 \& 17). Selectivity and specificity were well within the acceptance criteria for all analytes and IS.

\section{Cross validation to human \& pig plasma}

Following the validation of the assay for rat plasma, further, 1 day, 'fit for purpose' cross validation studies of the method were performed to enable the quantification of APAP and its metabolites in human and pig-derived plasma. In these cross validation studies, the method provided similar results to those obtained for rat plasma in terms of analytical figures of merit, carryover, recovery and matrix factors. The validation results are summarized in the appropriate sections of the supplementary data (see Supplementary Tables 18-23). In addition, the ability of the current method to perform the semi-quantitative analysis of PAP-G was investigated during the cross validation of the assay for pig plasma. In the absence of a stable isotope labeled IS for PAP-G validation for quantitative analysis was not attempted. The method was found to be linear over the concentration range measured $(16-500 \mu \mathrm{g} / \mathrm{ml})$ for PAP-G (see Supplementary Tables 20 \& 21). A representative chromatogram for pig plasma following the administration of APAP is shown in Supplementary Figure 2 and the application of the method to a pig hepatotoxicity study has recently been described [19]. 


\section{Mouse serum validation results}

A limited assessment of the assay, with the inclusion of APAP-OMe, for mouse serum was also made (details of standard curve concentrations etc., are summarized in Supplementary Tables 24-28). The limited 'fit for purpose' validation results obtained are provided in Supplementary Table 28 and show that the standard curves were linear, with $\mathrm{r}^{2}$ values above 0.99 , including the semiquantitative analysis of APAP-OMe over the range $0.64-20 \mu \mathrm{g} / \mathrm{ml}$. The assay appeared, based on the QC data, to be accurate and precise. Matrix factors were minimal with recoveries similar to plasma at over $80 \%$. Based on the partial validation performed here, we believe that the plasma method devised for these analytes can also be used, with caution, for mouse serum (see e.g., [20]).

Determination of acetaminophen \& metabolites in plasma following oral administration to the rat The assay was applied to the analysis of APAP, APAP-S, APAP-G, APAP-C, APAP-NAC and APAP-SG in the plasma of rats receiving either a single oral dose of the drug at either 0 (dose vehicle), 500 or $1500 \mathrm{mg} / \mathrm{kg}$. Neither APAP nor any of the targeted metabolites were detected in samples from predose time points or from vehicle-dosed control animals. The highest plasma concentrations of APAP were detected in samples obtained for the 1- and 2-h postdose time points for both the 500 and $1500 \mathrm{mg} / \mathrm{kg}$ dose groups. The APAP concentrations measured in these samples, apart from one sample from the $1500 \mathrm{mg} / \mathrm{kg}$ dose group, all fell between the upper and lower limits of quantification. However, this result was accepted as it was within "a value $25 \%$ above the ULOQ (i.e., ULOQ $\times 1.25$ )" as advocated by Bateman $e t$ al. in a recent publication [21].

Similarly, for APAP-S, the mean peak observed concentrations were obtained at the 1 - and 2-h time points and several samples, at both dose levels were at, or above, the ULOQ (Figure 3), although most of these were again within $25 \%$ of the ULOQ. For APAP-G, the mean peak plasma concentrations were seen for the 1- and 2-h postdose for the $500 \mathrm{mg} / \mathrm{kg}$ dose and slightly later, at the 2- and 4-h postdose time points, for the $1500 \mathrm{mg} / \mathrm{kg}$ dose level (Figure 3). In the case of the latter, the concentration in one sample exceeded the ULOQ but was considered as acceptable as it was also within $25 \%$ of the ULOQ.

The concentrations of the NAPQI-derived metabolites APAP-C, APAP-NAC and APAP-SG, were considerably lower than those of APAP and its phenolic sulphate and glucuronide conjugates. Both the APAP-C and APAP-NAC metabolites were detectable following APAP administration with peak observed concentrations seen at the 24-h time point at both doses. However, at early time points post administration, for both the 500 and $1500 \mathrm{mg} / \mathrm{kg}$ dose levels, the concentrations of the APAP-C metabolite were often at, or just below, the LLOQ (particularly for the $500 \mathrm{mg} / \mathrm{kg}$ dose group) (see Figure 4). However, while not quantifiable, these metabolites were clearly detectable and depending upon the purpose of the investigation could either be used to justify reanalysis of less diluted samples or for some other method of assessing the data. The reporting and use of values of a clearly detectable analyte that are below the LOQ has been (and continues to be) a matter of some debate as discussed in [22,23] and such a discussion is outside the scope of this study. However, it is evident that from the data presented in Figures $3 \& 4$, while the plasma concentrations of APAP and its phenolic conjugates APAP-G and APAP-S fall rapidly from their peak values at ca., 1-2-h postdose (Figure 3) those of the GSH-derived metabolites do not. So, the APAP-C and APAP-NAC metabolites were generally detectable in all of the postdose samples but were only reliably above the LLOQ at the later time points. Likewise, the low early time point concentrations of APAP-SG meant that, while detectable, the majority of the results, apart from the 24-h samples, fell below the LLOQ (Figure 4). The fact that these NAPQI-derived metabolites were detectable in the current analysis but were often at or below the LLOQ, reflects the fact that the samples were diluted 1 in 1000 prior to analysis in order that the more abundant analytes, such as APAP and the glucuronide and sulphate conjugates, were present in final concentrations within the linear range for their assay. In practice, if the three NAPQI-derived metabolites were the focus of the study, their accurate quantification could be readily achieved by analysis of a less dilute sample (as we have shown for mouse serum in a recent application of the method [20]).

The reconstructed ion mass chromatograms (Figure 5A \& B) for typical 1- and 24-h plasma samples for the $500 \mathrm{mg} / \mathrm{kg}$ dose show the change in the balance of metabolites within the profile with time after dosing.

As noted in the introduction, the relatively high concentrations of the drug and its major metabolites present in plasma and serum following APAP and administration mean that, in practice, they are amenable to analysis by a wide range of methods, including LC-UV [8-10]. However, the development of HPLC-MS-based methods for the quantification of APAP and metabolites offers benefits in terms of increased specificity. This increased specificity combined with greatly increased sensitivity, results in greater efficiency by enabling the adoption of minimal sample preparation methods and shorter analysis times as well as the use of much smaller samples. As indicated previously, 

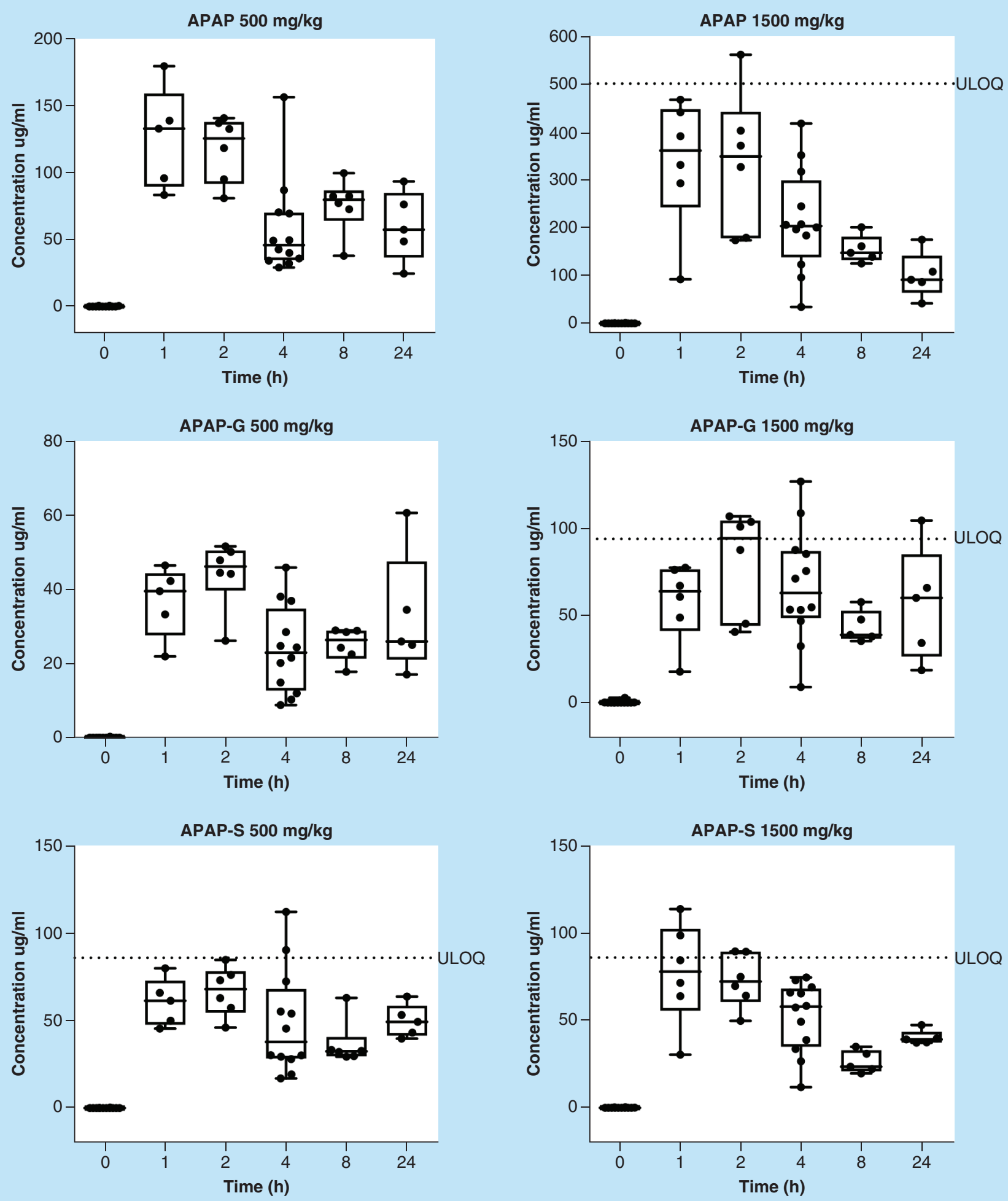

Figure 3. Plasma concentration data for APAP, APAP-G and APAP-S, obtained predose and at various time points up to 24-h postdose to rats administered APAP at either 500 or $1500 \mathrm{mg} / \mathbf{k g}$ (based on the analysis of $5 \mu \mathrm{l}$ of sample). Each point represents an individual animal, boxes represent the median and interquartile range and whiskers show the full range. 

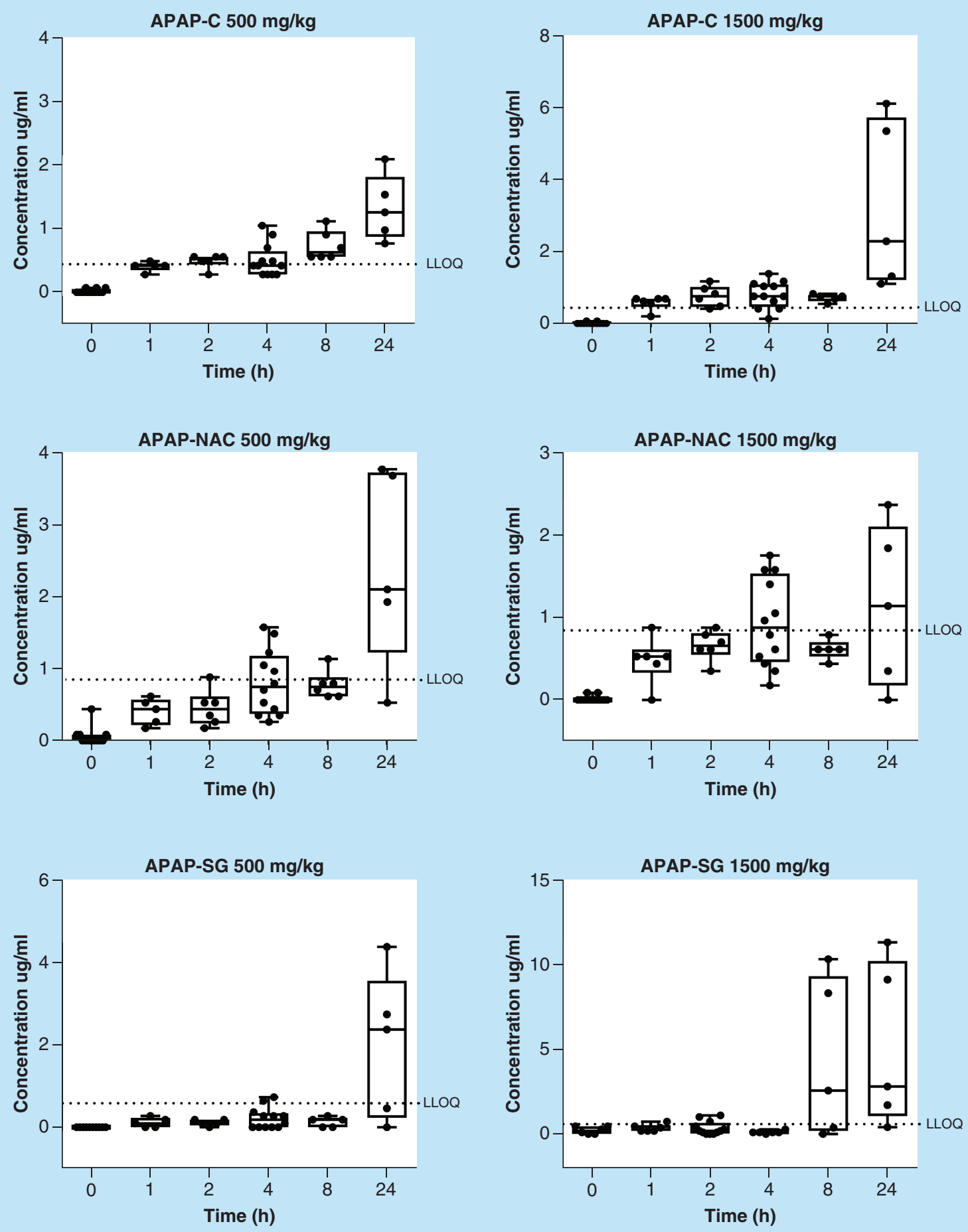

Figure 4. Plasma concentration data for APAP-C (upper), APAP-NAC (middle) and APAP-SG (lower) conjugates, at various time points up to $24-\mathrm{h}$ postdose, obtained from rats administered APAP at either 500 or $1500 \mathrm{mg} / \mathrm{kg}$. Each point represents an individual animal, boxes represent the median and interquartile range whiskers show the full range. 
(A)

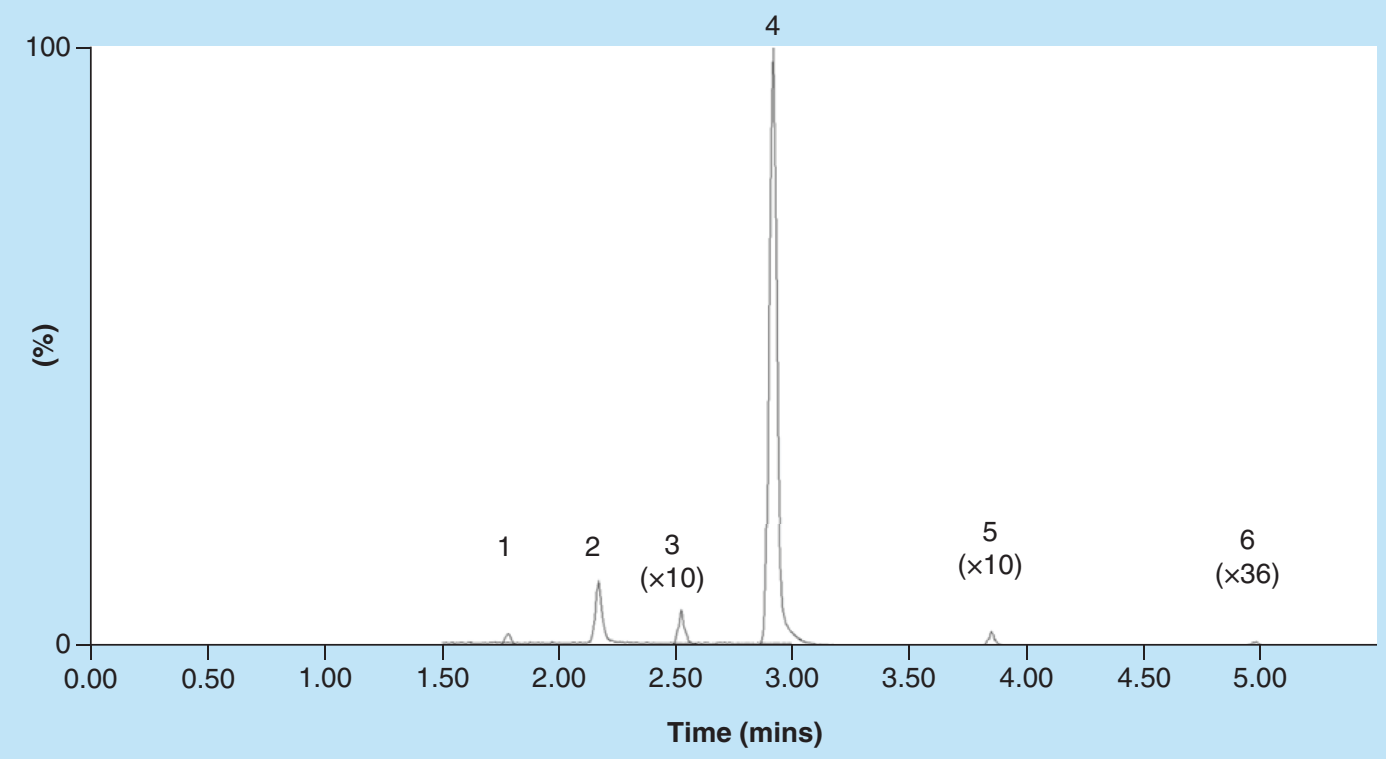

(B)

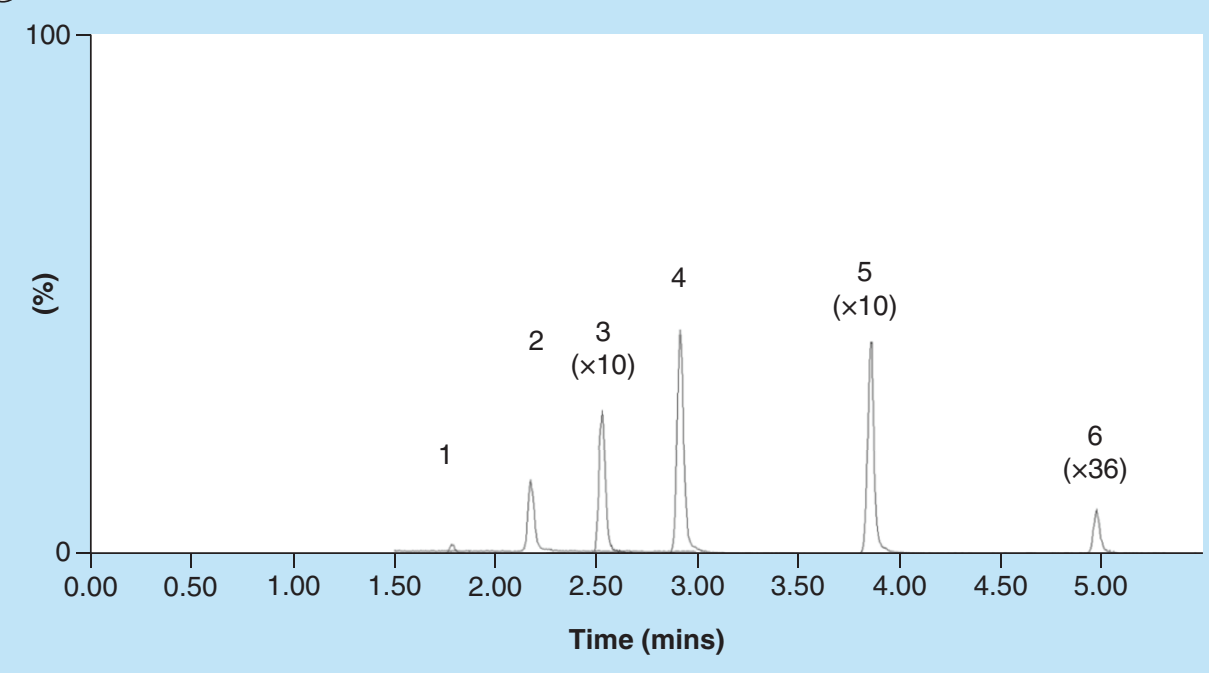

Figure 5. Representative ion mass chromatograms for samples from the $500 \mathrm{mg} / \mathrm{kg}$ APAP dose group. (A) One hour postdose or (B) 24-h postdose. Some chromatographic peaks have been increased in intensity in some cases for clarity (as indicated by the numbers in parenthesis above the peaks). All chromatographic peak heights are relative to APAP in Figure 5A. 1 = APAP-G, 2 = APAP-S, 3 = APAP-C, 4 = APAP, 5 = APAP-SG, 6 = APAP-NAC.

several pre-existing LC-MS-based assays for APAP and (a variable number of) metabolites have been described for use in biofluids such as urine and blood-derived samples [11-17]. Some of these methods, such as that developed for mouse urine [11], are limited in their coverage to the determination of APAP and the major conjugated metabolites APAP-G and APAP-S. That method, which used the structural analog 3-acetamidophenol as an IS, employed an isocratic reversed-phase separation with a nominal run time of $10 \mathrm{~min}$. However, with column washing and re-equilibration, the overall analysis time per sample was $30 \mathrm{~min}$. Subsequent methods enabled the determination of APAP and either APAP-G [12], or both APAP-G and APAP-S [13]. The analysis of APAP and APAP-G [12] used isocratic reversed-phase HPLC-MS/MS and was developed for the quantification of these analytes in human plasma and urine [12]. Similarly, an isocratic reversed-phase LC-MS/MS assay was used to determine APAP, APAP- 
G and APAP-S in mouse plasma [13], with APAP-d4 as sole IS, with an overall analysis time of $10 \mathrm{~min} / \mathrm{sample}$ [13]. More recent methods have described the analysis of plasma or urine samples with a more comprehensive coverage of APAP and its metabolites, including those resulting from the production of NAPQI [14-17]. The first of these methods allowed the determination of APAP and six metabolites (APAP-G, APAP-S, APAP-OMe, APAP-NAC, APAP-C and APAP-SG) in rat plasma using HPLC-MS/MS [14]. This assay used deuterated APAP and APAP-G as ISs and employed a reversed-phase gradient for separation to give a run time of $16 \mathrm{~min} / \mathrm{sample}$. However, two runs were required to obtain the required data as the method employed positive ESI for APAP and 3-methoxy-APAP (APAP-OMe) and negative ESI for APAP-S, APAP-G, APAP-SG, APAP-NAC and APAP-C. The validated assay had an LLOQ of $100 \mathrm{ng} / \mathrm{ml}$ for APAP, APAP-S, APAP-G and for $10 \mathrm{ng} / \mathrm{ml}$ APAP-SG, APP-NAC, APAP-C and APAP-OMe. Another multimetabolite method, also based on gradient reversed-phase HPLC-MS/MS quantified APAP, APAP-G, APAP-S, APAP-NAC, APAP-C and APAP-SG, using APAP-d4 and APAP-S-d3 as ISs. This method analyzed $10 \mu \mathrm{l}$ of samples of both human urine and plasma with an analysis time of $20 \mathrm{~min} / \mathrm{sample}$ [15].

More recently, a method based on reversed-phase gradient HPLC-MS/MS, with a total run time of 9 min, was developed to analyze APAP, APAP-G, APAP-S, APAP-OMe, APAP-SG, APAP-C and APAP-NAC in $100 \mu$ of human plasma [16]. A feature of this method was the use of APAP-d4, APAP-G-d3, APAP-S-d3, APAP-C-d5 and APAP-NAC-d5 to monitor the assay, rather than a reliance on a more limited number of ISs. A second method, this time based on UHPLC-MS also employed reversed-phase gradient chromatography, with a rapid (4.5 min) separation, to analyze APAP, APAP-G, APAP-S, APAP-C, APAP-SG and APAP-NAC. In addition, protein-derived APAP-C formed via the reaction of NAPQI with protein was also quantified. The method was applied to the analysis of $10 \mu \mathrm{l}$ of human plasma obtained from children taking part in a pediatric clinical study [17]. APAP-d3 was used as the IS for all analytes in this method.

The U(H)PLC-MS method described here has been developed for the analysis of small $(5 \mu \mathrm{l})$ samples of rat, human and pig plasma making it suitable for studies, in animals or patients, where only limited quantities of plasma or serum are available. Unlike some earlier methods, the assay employs stable isotopically labeled ISs for all the analytes validated for quantitative analysis (APAP, APAP-G, APAP-S, APAP-C, APAP-SG and APAP-NAC). The method has a short analysis time $(7.5 \mathrm{~min} /$ sample) and requires minimal sample preparation, offering the potential for efficient large-scale sample analysis.

In addition to the quantification of APAP and the metabolites described above, the method can be used to monitor, and provide semi-quantitative data, for the minor 3-methoxy-metabolite (for which no isotopically labeled IS was available) using an external standard curve. Similarly, the metabolite PAP-G (formed by the glucuronidation of $p$-aminophenol produced by the $N$-deacetylation of APAP and subsequent $\mathrm{O}$-glucuronidation) can also be monitored, and semi-quantified, in for example, porcine plasma using a similar approach. The absence of a stable isotope labeled IS for these analytes makes meaningful validation problematic. However, we consider the use of such external standard curves for semi-quantitative analysis to enable monitoring both feasible and, in our opinion, preferable to using the stable isotope labeled analog of either another metabolite, or APAP itself, as a 'surrogate' IS. For a rapid and fit for purpose assessment of exposure to these, potentially important metabolites, we believe this to be an appropriate response to the absence of suitable labeled analytes. However, stable isotope-labeled standards of these metabolites will hopefully become available in due course to enable methods for absolute quantification to be developed.

\section{Conclusion}

A 'fit for purpose' rapid and sensitive U(H)PLC-MS/MS method for the quantitative analysis of acetaminophen and five of its metabolites (APAP-G, APAP-S, APAP-C, APAP-SG and APAP-NAC) using deuterated ISs, has been developed for the analysis of plasma samples from rat, pig and human, and serum from mouse. In addition, a further two metabolites APAP-OMe and PAP-G can be monitored and semi-quantified using external standards. The method, which requires only $5 \mu \mathrm{l}$ of sample, has been validated for use in rat plasma and cross validated for human and pig plasma and mouse serum.

\section{Future perspective}

APAP remains a major cause of hepatic toxicity and liver transplantation in humans. While there has been much progress in understanding the mechanism of hepatotoxicity, there remains the need to obtain improved methods for predicting liver failure or recovery. The toxicity is thought to be driven by the metabolism of the drug, particularly by CYP2E1. Sensitive and specific methods for the determination of the drug and its metabolites remain important 
for studying the drugs toxicity and finding suitable biomarker combinations. As such, assays for the analysis of the biofluids of animal models and humans will remain important in strategies designed to improve patient outcomes in the case of overdose etc.

\section{Summary points}

\section{Background}

- To fully understand the factors resulting in APAP hepatotoxicity, the determination of the drug and its metabolites is important.

- For this reason, a rapid and sensitive analysis, providing a fit for purpose UPLC-MS method for acetaminophen (paracetamol, APAP) and seven metabolites in plasma or serum, was developed.

\section{Experimental}

- The developed method enabled quantification of APAP and its sulphate, glucuronide, glutathione, cysteinyl and $\mathrm{N}$-acetylcysteinyl conjugates using stable isotope-labeled internal standards.

- P-Aminophenol glucuronide and APAP-OMe were monitored and semi-quantified with external standards.

Results \& discussion

- A simple and rapid method, with minimal sample preparation and using $5 \mu$ of sample was devised and applied to rat plasma samples.

- In the case of rat plasma, the method enabled the detection of APAP and its conjugates following oral administration of 500 or $1500 \mathrm{mg} / \mathrm{kg}$ for up to 24-h postdose.

- The method has also shown utility for the analysis of mouse serum and pig plasma (where, in the latter, $p$-aminophenol glucuronide was found to be a major circulating metabolite).

\section{Conclusion}

- The method has good sensitivity and is suitable for the determination of the targeted analytes in the plasma of rat, pig and humans and mouse serum.

Financial \& competing interests disclosure

I Grant was funded through a Medical Research Council (MRC) ITTP PhD studentship and R Dargue was funded via a BBSRC Case Studentship (GSK). The NIHR Imperial BRC is acknowledged for support to M Coen. The authors have no other relevant affiliations or financial involvement with any organization or entity with a financial interest in or financial conflict with the subject matter or materials discussed in the manuscript apart from those disclosed.

No writing assistance was utilized in the production of this manuscript.

\section{Ethical conduct of research}

The authors state that they have obtained appropriate institutional review board approval for all animal experimental investigations which were conducted in compliance with UK home office licences issued under the UK animals (Scientific Procedures) Act 1986.

\section{Open access}

This work is licensed under the Creative Commons Attribution 4.0 License. To view a copy of this license, visit http://creativecommons.org/licenses/by/4.0/

\section{References}

Papers of special note have been highlighted as: $\bullet$ of interest or $\bullet \bullet$ of considerable interest

1. Makin AJ, Wendon J, Williams R. A 7-year experience of severe acetaminophen-induced hepatotoxicity. Gastroenterology 109(6), 1907-1916 (1995).

2. Larson AM, Polson J, Fontana RJ et al. Acetaminophen-induced acute liver failure: results of a United States multicenter, prospective study. Hepatology 42(6), 1364-1372 (2005).

3. G Ostapowicz G, Fontana RJ, Schiodt FV et al. Larson Results of a prospective study of acute liver failure at 17 tertiary care centers in the United States. Ann. Intern. Med. 137(12), 947-954 (2002).

4. Larson AM, Polson J, Fontana RJ et al. Acetaminophen sets records in the United States: number 1 analgesic and number 1 cause of acute liver failure acetaminophen-induced acute liver failure: results of a United States multicenter, prospective study. Hepatology 42(4), 1364-1372 (2005).

5. Myers RP, Li B, Shaheen AA. Emergency department visits for acetaminophen overdose: a Canadian population-based epidemiologic study (1997-2002). CJEM 9(4), 267-274 (2007).

6. Dahlin DC, Miwa GT, Lu AY, Nelson SD. N-acetyl-p-benzoquinone imine: a cytochrome P-450-mediated oxidation product of acetaminophen. Proc. Natl Acad. Sci. USA 81(5), 1327-1331 (1984).

7. Mitchell JR, Jollow DJ, Potter WZ, Gillette JR, Brodie BB. Acetaminophen-induced hepatic necrosis. IV. Protective role of glutathione. J. Pharmacol. Exp. Ther. 187(1), 211-217 (1973). 
8. Di Girolamo A, O'Neill WM, Wainer IW. A validated method for the determination of paracetamol and its glucuronide and sulphate metabolites in the urine of HIV+/AIDS patients using wavelength switching UV detection. J. Pharm. Biomed. Anal. 17(6-7), 1191-1197 (1998).

9. Vertzoni MV, Archontaki P, Galanopoulou P. Development and optimization of a reversed-phase high-performance liquid chromatographic method for the determination of acetaminophen and its major metabolites in rabbit plasma and urine after a toxic dose. J. Pharm. Biomed. Anal. 32(3), 487-493 (2003).

10. Jensen LS, Valentine J, Milne RW, Evans AM. The quantification of paracetamol, paracetamol glucuronide and paracetamol sulphate in plasma and urine using a single high-performance liquid chromatography assay. J. Pharm. Biomed. Anal. 34(3 ), 585-593 (2004).

11. Hweavitharana AK, Lees S, Dawson PA, Markowich D, Shaw PN. Development of an HPLC-MS/MS method for the selective determination of paracetamol metabolites in mouse urine. Anal. Biochem. 374(1), 106-111 (2008).

12. Tan QY, Zhu RH, Li HD, Wang F, Yan M, Dai LB. Simultaneous quantitative determination of paracetamol and its glucuronide conjugate in human plasma and urine by liquid chromatography coupled to electrospray tandem mass spectrometry: application to a clinical pharmacokinetic study. J. Chromatogr. B 893-894, 162-167 (2012).

13. Gicquel T, Aubert JT, Lepage S, Fromenty B, Morel I. Quantitative analysis of acetaminophen and its primary metabolites in small plasma volumes by liquid chromatography-tandem mass spectrometry. J. Anal. Toxicol. 37(2),110-116 (2013).

14. An JH, Lee HJ, Jung BH. Quantitative analysis of acetaminophen and its six metabolites in rat plasma using liquid chromatography/tandem mass spectrometry. Biomed. Chromatogr. 26(12), 1596-1604 (2012).

- A useful method that describes a HPLC-based method for analysis of APAP and metabolites.

15. Cook SF, King AD, van den Anker JN, Wilkins DG. Simultaneous quantification of acetaminophen and five acetaminophen metabolites in human plasma and urine by high-performance liquid chromatography-electrospray ionization-tandem mass spectrometry: method validation and application to a neonatal pharmacokinetic study. J. Chromatogr. B 1007, 30-42 (2015).

16. Lammers LA, Achterbergh RM, Pistorius CM, Romijn JA, Mathôt RA. A Quantitative method for simultaneous analysis of acetaminophen and 6 metabolites. Ther. Drug Monit. 39(2), 172-179 (2017).

17. Flint RB, Mian P, van der Nagel B, Slijkhuis N, Koch BC. Quantification of acetaminophen and its metabolites in plasma Using UPLC-MS: doors open to therapeutic drug monitoring in special patient populations. Ther. Drug Monit. 39 164-171 (2017).

- A useful UHPLC method for APAP and metabolites in plasma.

18. US FDA. Bioanalytical Method Validation Guidance for Industry (2001). http://www.fda.gov

19. Dargue R, Zia Lau C et al. Metabolism and effects on endogenous metabolism of paracetamol (acetaminophen) in a porcine model of liver failure. Toxicol. Sci. 10.1093/toxsci/kfaa023 (2020) (Epub ahead of print).

20. Triantafyllou E, Pop OT, Possamai LA et al. MerTK expressing hepatic macrophages promote the resolution of inflammation in acute liver failure. Gut 67(2), 333-347 (2018).

21. Bateman KP, Cohen L, Emary B, Pucci V. Standardized workflows for increasing efficiency and productivity in discovery stage bioanalysis. Bioanalysis 5(14), 1783-1794 (2013).

-• Provides guidance on fit for purpose methodology, including assessment of concentration above the upper LOQ, when strict adherence to regulatory guidance is not necessarily appropriate.

22. Senn S, N Holford N, Hockey H. The ghosts of departed quantities: approaches to dealing with observations below the limit of quantification. Stat. Med. 31(30), 4280-4295 (2012).

-• Guidance on the assessment of analytes detected but below the LOQ.

23. Duggan JX. Quantification below the LLOQ in regulated LC-MS/MS assays: a review of bioanalytical considerations and cautions. Bioanalysis 11(8), 797-814 (2019).

-. Guidance on the assessment of analytes detected but below the LOQ. 